\title{
Relationship of blood rheology to lipoprotein profile during normal pregnancies and those with intrauterine growth retardation
}

\author{
A Muñoz, J Uberos, A Molina, A Valenzuela, D Cano, C Ruiz, J A Molina Font
}

\begin{abstract}
Aims-The effects on fetal growth of hyperlipidaemia in pregnancy are not well understood at present. In this study the different lipid fractions in normal pregnancies and pregnancies complicated by intrauterine growth retardation (IUGR) were determined and related to changes in plasma and serum viscosity.

Methods-Two groups of pregnant women were studied. Group 1 consisted of 35 healthy pregnant women aged between 21 and 38 years with no previous pathology and a normal pregnancy to term. Group 1 patients were studied at four periods defined at the start of the study: (1) $\leq 17$ weeks; (2) 18-24 weeks; (3) 25-32 weeks, (4) $\geq 33$ weeks. Group 2 consisted of 24 pregnant women aged between 16 and 34 years with ultrasound diagnosed IUGR confirmed after birth. Plasma lipids and plasma and serum viscosity were measured. Results-Plasma triglycerides, low density lipoprotein cholesterol, and total cholesterol increased progressively throughout pregnancy, with significantly higher values after week 25. Apolipoprotein A (ApoA) and triglyceride concentrations were significantly lower in the IUGR group than in the normal group. The HDLAApoA ratio was greater in the IUGR group than in the control group, as was the ApoB/ ApoA ratio. There were no differences in the other lipids. Plasma and serum viscosity was higher in the IUGR group than in the normal group.

Conclusions-Haemorheological modifications in the IUGR group are partly secondary to changes in high density lipoprotein metabolism and the competitive inhibition of fibrinolysis by ApoB, which is increased in pregnancies with IUGR. Changes in ApoA, and more specifically in the ApoB/ApoA ratio, could be good markers for the early detection of IUGR. ( $f$ Clin Pathol 1995;48:571-574)
\end{abstract}

Keywords: Intrauterine growth retardation, hyperlipidaemia, fibrinolysis, fibrinogen, pregnancy, viscosity, blood rheology.

During pregnancy, increases in female sex hormones and lipolysis due to placental lactogen cause an increase in lipid synthesis and lipid metabolism. This in turn leads to increased cholesterol, triglyceride, and phospholipid concentrations.
Hyperlipidaemia is commonly found in the normal population associated with pathologies such as atherosclerosis and hypertension. In pregnant women, however, it also has serious implications but these are not yet fully understood. The increased nutritional requirements of the fetoplacental unit and the endocrine profile during pregnancy could provide the starting point to explain these modifications. ${ }^{1}$

More studies on biochemical changes during pregnancy are necessary to establish a range of normality in order to characterise excessive gestational lipolytic responses. This would enable us to determine if the changes in the lipoprotein profile during pregnancy imply a greater atherogenic risk with possible repercussions on fetal growth and development. In this study the different lipid fractions in normal pregnancies and in pregnancies with intrauterine growth retardation (IUGR) were determined and related to changes in plasma and serum viscosity.

\section{Methods}

SAMPLE DEFINITION AND CHARACTERISTICS

Fifty nine pregnant women were studied. These were divided into two groups. The first group consisted of 35 healthy pregnant women aged between 21 and 38 years (27(SD 3.5) years) with no previous pathology, a pregnancy to term without complications (with a gestational age of $279(10)$ days), and a birthweight of 3345.4(449.3)g. The second group consisted of 24 pregnant women aged between 16 and 34 years $(24.5$ (4.9) years) with a gestational age at term of $278(12)$ days, with ultrasound diagnosed IUGR. The diagnosis of IUGR was made on the basis of a biparietal diameter $>2$ SD below the mean at 33 weeks of gestational age. ${ }^{2}$ The birthweight in newborns with intrauterine growth retardation was between $1170 \mathrm{~g}$ and $2830 \mathrm{~g}(2305 \cdot 4(544 \cdot 7) \mathrm{g})$.

\section{METHODOLOGY}

The women were fasting before blood was drawn. Analytical determinations were carried out in four study periods defined at the beginning of the study: (1) pregnant $\leq 17$ weeks; (2) pregnant 18 to 24 weeks; (3) pregnant 25 to 32 weeks; (4) pregnant $\geq 33$ weeks. The following variables were analysed in all cases: total cholesterol, triglycerides, high density lipoproteins (HDL), low density lipoproteins (LDL), free fatty acids, and phospholipids by enzymatic microtechnique, ${ }^{3-7}$ apolipoprotein A 
Table 1 Apolipoprotein and lipid means in healthy pregnant controls and in women with intrauterine growth retardation. Values are means (SD)

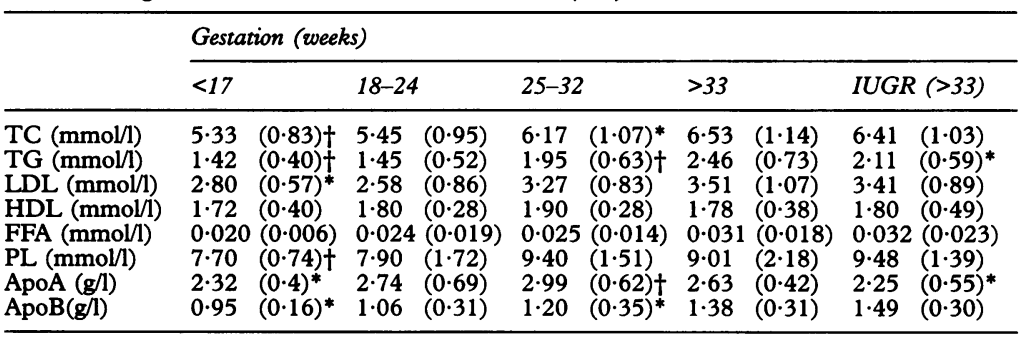

IUGR = intrauterine growth retardation over 33 weeks of gestational age; ApoA=apolipoprotein $\mathrm{A} ; \mathrm{ApoB}=$ apolipoprotein $\mathrm{B} ; \mathrm{TC}=$ total cholesterol; $\mathrm{TG}=$ triglycerides; $\mathrm{HDL}=$ high density lipoprotein cholesterol; $\mathrm{LDL}=$ low density lipoprotein cholesterol; FFA = free fatty acid; PL= phospholipid.

${ }^{*} \mathrm{p}<0.05 ;+\mathrm{p}<0.01$, statistical significance observed among women at $>33$ weeks' gestation, and those at $<17,18-24,25-32$ weeks' gestation and IUGR ( $>33$ weeks).

Table 2 Ratios of lipoprotein constituents in healthy pregnant controls and in women with intrauterine growth retardation. Values are means (SD)

\begin{tabular}{|c|c|c|c|c|c|}
\hline & \multicolumn{5}{|c|}{ Gestation (weeks) } \\
\hline & $<17$ & $18-24$ & $25-32$ & $>33$ & IUGR (>33) \\
\hline $\begin{array}{l}\text { ApoB/ApoA } \\
\text { TC/HDL } \\
\text { LDL/HDL } \\
\text { HDL/ApoA } \\
\text { FFA/TG }\end{array}$ & $\begin{array}{ll}0.40 & (0.49)^{*} \\
3.09 & (0.69) \\
1.62 & (0.53) \\
0.74 & (0.05) \\
0.014 & (0.002)\end{array}$ & $\begin{array}{ll}0.38 & (0.45)^{*} \\
3.02 & (0.56) \dagger \\
1.43 & (0.49) \\
0.65 & (0.07) \\
0.016 & (0.005)\end{array}$ & $\begin{array}{l}0.40 \quad(0.56)^{*} \\
3.24 \quad(0.85)^{*} \\
1.72 \quad(0.57) \\
0.63(0.05) \\
0.012(0.002)\end{array}$ & $\begin{array}{ll}0.52 & (0.74) \\
3.66 & (0.90) \\
1.97 & (0.82) \\
0.67 & (0.05) \\
0.012 & (0.002)\end{array}$ & $\begin{array}{ll}0.66 & (0.24) \dagger \\
3.56 & (1.27) \\
1.89 & (1.05) \\
0.80 & (0.1) \dagger \\
0.015 & (0.002)\end{array}$ \\
\hline
\end{tabular}

IUGR = intrauterine growth retardation over 33 weeks of gestational age; ApoA = apolipoprotein $\mathrm{A} ; \mathrm{ApoB}=$ apolipoprotein $\mathrm{B}$; $\mathrm{TC}=$ total cholesterol; $\mathrm{TG}=$ triglycerides; HDL $=$ high density lipoprotein cholesterol; $\mathrm{LDL}=1 \mathrm{c}$ density lipoprotein cholesterol; FFA = free fatty acid; $\mathrm{PL}=$ phospholipid.

${ }^{*} \mathrm{p}<0.05 ;+\mathrm{p}<0.01$, statistical significance observed among women at $>33$ weeks' gestation, and those at $<17,18-24,25-32$ weeks' gestation and IUGR ( $>33$ weeks).

Table 3 Plasma proteins and serum and plasma viscosity in healthy pregnant controls and in women with intrauterine growth retardation. Values are means (SD)

\begin{tabular}{|c|c|c|c|c|c|}
\hline & \multicolumn{5}{|c|}{ Gestation (weeks) } \\
\hline & $<17$ & $18-24$ & $25-32$ & $>33$ & IUGR (>33) \\
\hline $\begin{array}{l}\text { Albumin }(\mathrm{g} / \mathrm{dl}) \\
\alpha_{1} \text { globulin }(\mathrm{g} / \mathrm{dl}) \\
\alpha_{2} \text { globulin }(\mathrm{g} / \mathrm{dl}) \\
\beta \text { globulin }(\mathrm{g} / \mathrm{dl}) \\
\gamma \text { globulin }(\mathrm{g} / \mathrm{dl}) \\
\text { Total protein }(\mathrm{g} / \mathrm{dl})\end{array}$ & $\begin{array}{l}4.34(0.56) \dagger \\
0.32(0.05) \\
0.68(0.1) \\
0.95(0.27) \\
0.81(0.16) \\
7.12(0.7) \dagger\end{array}$ & $\begin{array}{l}3.90(0.41) \dagger \\
0.28(0.04)^{*} \\
0.67(0.13) \\
0.90(0.21)^{*} \\
0.76(0.19) \\
6.53(0.58)\end{array}$ & $\begin{array}{l}3.66(0.34) \\
0.31(0.05) \\
0.71(0.13) \\
1.03(0.13) \\
0.80(0.21) \\
6.53(0.48)\end{array}$ & $\begin{array}{l}3.52(0.29) \\
0.33(0.04) \\
0.72(0.13) \\
1.02(0.18) \\
0.71(0.17) \\
6.31(0.50)\end{array}$ & $\begin{array}{l}3.46(0.43) \\
0.42(0.19) \dagger \\
0.76(0.22) \\
0.99(0.21) \\
0.79(0.30) \\
6.39(0.74)\end{array}$ \\
\hline $\begin{array}{l}\text { Plasma viscosity } \\
(\mathrm{PV})(\mathrm{mPa} \cdot \mathrm{s})\end{array}$ & $1.09(0.08)$ & $1.06(0 \cdot 15)$ & $1.08(0 \cdot 15)$ & $1 \cdot 12(0 \cdot 12)$ & $1.26(0.15)^{*}$ \\
\hline $\begin{array}{l}\text { Serum viscosity } \\
\text { (SV) (mPa } \mathrm{s})\end{array}$ & $0.95(0.09)$ & $0.96(0 \cdot 13)$ & $1 \cdot 00(0 \cdot 12)$ & $0.94(0.07)$ & $1.03(0.13)^{*}$ \\
\hline PV-SV (mPa·s) & $0 \cdot 13(0 \cdot 13)$ & $0.09(0.2)$ & $0.08(0 \cdot 14)$ & $0 \cdot 17(0 \cdot 15)$ & $0.22(0.2)$ \\
\hline
\end{tabular}

IUGR = intrauterine growth retardation over 33 weeks of gestational age; PV-SV=difference between plasma and serum viscosity.

${ }^{*} \mathrm{p}<0.05 ;+p<0.01$, statistical significance observed among women at $>33$ weeks' gestation, and those at $<17,18-24,25-32$ weeks' gestation and IUGR ( $>33$ weeks).

(ApoA) and apolipoprotein B (ApoB) by simple radial immunodiffusion. ${ }^{8}$ Systolic and diastolic blood pressures were recorded for every case in each study period; pressures were measured three times with the women resting supine, and the mean of the three values was used. Plasma and serum viscosities were measured at $37^{\circ} \mathrm{C}$ with a Harkness capillary viscosimeter (Coulter Electronics). Statistical analysis included the Shapiro and Wilk's normality test, one way variance analysis (ANOVA), a comparison of means, and Pearson's " $r$ " correlation study.

\section{Results}

The means and standard deviations of all the lipid measurements analysed during pregnancy are given in table 1. Total cholesterol increased progressively during pregnancy and the in- crease was significant after week 25 . Triglycerides and LDL also increased, with significantly higher values in period 4 compared with period $1(t=4.61, \mathrm{p}<0.01$; and $t=2 \cdot 17$, $\mathrm{p}<0.05)$ and with period $2(t=6.07, \mathrm{p}<0.01$; and $t=2.51, \mathrm{p}<0.05)$. HDL and free fatty acids did not change significantly during pregnancy $(F=1 \cdot 16, p=N S ;$ and $F=1 \cdot 63$, $\mathrm{p}=\mathrm{NS}$ ). The phospholipids increased until period 3 and then stabilised and decreased slightly. ApoA increased up to period 3 after which it decreased significantly $(t=2 \cdot 61$, $\mathrm{p}<0 \cdot 05)$. ApoB increased gradually throughout pregnancy.

Systolic blood pressure did not change significantly during pregnancy $(\mathrm{F}=1 \cdot 67, \mathrm{p}=\mathrm{NS})$. However, diastolic blood pressure was greater in periods 2 and 3 than in period $1(t=2 \cdot 47$, $\mathrm{p}<0.05$; and $t=2.53, \mathrm{p}<0.05$ respectively). At no time during pregnancy were the blood pressure values abnormal in the IUGR group.

In period 1 of the normal pregnancies systolic blood pressure was found to be correlated with total cholesterol $(r=0.33, p<0.05), L D L$ $(\mathrm{r}=0.53, \mathrm{p}<0.01)$, free fatty acids $(\mathrm{r}=-0.42$, $\mathrm{p}<0.05)$, and phospholipids $(\mathrm{r}=-0.34$, $\mathrm{p}<0.05)$. The diastolic blood pressure was correlated with triglycerides $(\mathrm{r}=-0.34, \mathrm{p}<0.05)$ and phospholipids $(\mathrm{r}=-0.51, \mathrm{p}<0.01)$. In period $3 \mathrm{HDL}$ and systolic blood pressure were correlated $(r=-0.36, p<0.05)$ and diastolic blood pressure was correlated with total cholesterol $(r=0.42, p<0.05)$. In period 4 there was no significant relationship between the blood pressure and the lipid variables.

After 33 weeks the ApoA and triglyceride concentrations were significantly lower in the IUGR group than in the controls, although no differences were observed with the other lipids (tables 1 and 2). Pregnant women with IUGR had similar total protein values to the control group, with the exception of $\alpha_{1}$ globulin which was higher in the IUGR group (table 3 ). Relationships between HDL and ApoA differed between the groups: the IUGR group had a highly atherogenic HDL/ApoA ratio, significantly greater than that of the control group (table 2).

Table 3 shows that there was significantly higher plasma and serum viscosity in the IUGR group than the values recorded in the same periods in the normal pregnancies. The difference between these two variables is an indirect method of calculating the viscosity effect of the fibrinogen fraction. 9 The difference between the viscosities in period 4 of the control group, $0.17(\mathrm{SD} 0.15) \mathrm{mPa} . \mathrm{s}$, is not significantly different to that of the IUGR group, $0.22(0.20) \mathrm{mPa} . \mathrm{s}(t=1.05, \mathrm{p}=\mathrm{NS})$. The coefficients of variation for the control and IUGR group viscosity differences were $88.2 \%$ and $71.4 \%$, respectively.

\section{Discussion}

Changes in lipid levels during pregnancy result from the metabolic adaptation of the mother. Mobilisation of fat deposits, the increase in free fatty acids, and the relationship between circulating progesterone and oestrogen levels ${ }^{10}$ 
suggest that these hormones are responsible for the lipid changes observed. Placental lactogen favours the release of fats from their deposits and an increase in free fatty acids, both being vital adaptations to ensure the availability of adequate energy substrate to the fetus. ${ }^{1}$ Like Jimenez et $a l,{ }^{11}$ we did not find any significant differences in HDL during pregnancy, although a slight increase in mean HDL levels was observed between weeks 25 and 32 as also reported by Piechota and Staszewski. ${ }^{10}$ Increases in total cholesterol and triglycerides, confirmed by other workers, ${ }^{1012-14}$ are observed in the last few weeks of pregnancy. The small nonsignificant increase in free fatty acid concentrations throughout pregnancy suggests that lipolysis of endogenous triglycerides is not increased. In accordance with other investigators, ${ }^{1015}$ ApoB concentrations progressively increase throughout pregnancy. In normal pregnancies, mean ApoA values show a non-significant decrease after week 33 whereas in the women with IUGR a decrease to below pregestational levels is observed (table 1).

An increase in lipid levels after week 25 of pregnancy is referred to in more than one paper. ${ }^{1013}$ This results from adaptation of lipid metabolism to the requirements of pregnancy. The increase in triglyceride concentration in normal pregnancies indicates a progressive increase in the supply of free fatty acids to the fetus during pregnancy. The free fatty acids to triglyceride ratio, indicative of triglyceride lipolysis, decreases slightly after week 25 , in spite of an increase in triglyceride con-
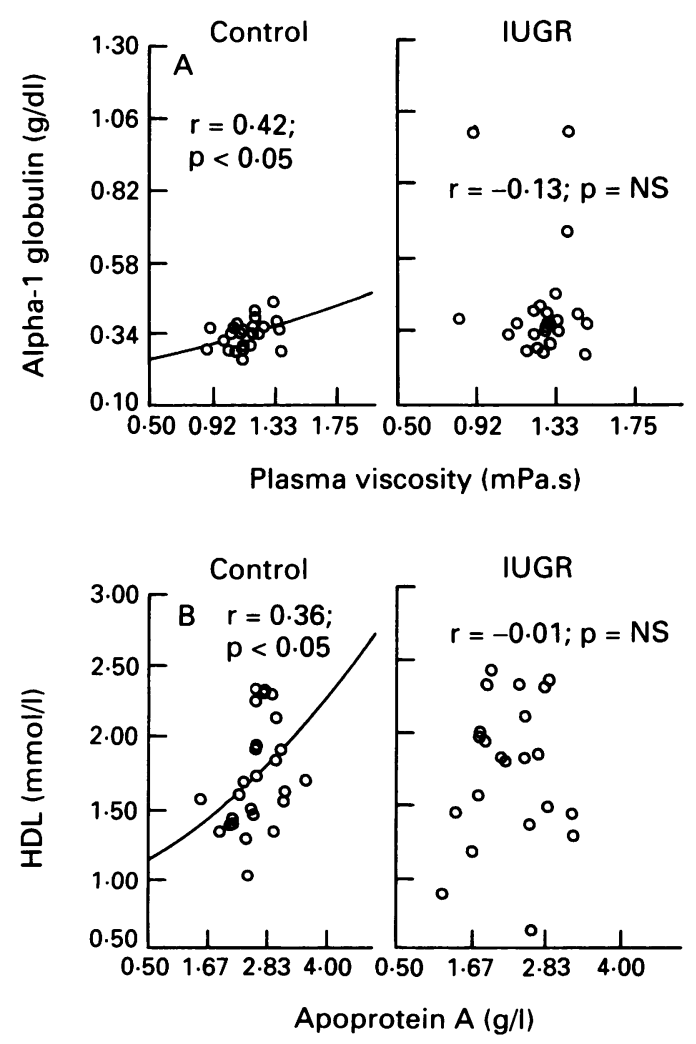

Correlation and regression between: (A) $\alpha_{1}$ globulin and plasma viscosity in pregnant healthy controls (control) and in women with intrauterine growth retardation (IUGR); (B) Apolipoprotein constituents and high density lipoprotein. centrations in the last period of pregnancy. This suggests that the increase in endogenous triglycerides is not equivalent to the increase in endogenous lipolysis (table 2).

The ApoA concentration increases significantly between weeks 25 and 32 of pregnancy, although the HDL/ApoA ratio was not significantly different in the gestational periods considered. Pregnant women with IUGR, however, have modifications in HDL composition (a significantly greater HDLApoA ratio). In contrast to the findings of Piechota and Staszewski, ${ }^{10}$ we could not detect any significant changes in HDL composition in the final gestational stages in the normal pregnancies; this is probably because these investigators did not distinguish between normal pregnancies and pregnancies with IUGR, which in our study are treated as two separate groups.

The ApoB/ApoA ratio is considered to be the most sensitive atherogenic index and its mean value at the end of the pregnancy is significantly higher than in previous stages. This indicates that the lipoprotein profile in normal pregnancies is more atherogenic in the final stages and is significantly abnormal in women with IUGR (table 2).

The LDL and HDL concentrations in normal pregnancies are closely related to circulating progesterone and oestrogen and to the pregnancy period in which they are determined. The HDL concentration increases, through a rise in ApoA synthesised predominantly in the liver, when oestrogen levels are raised. ${ }^{10}$ The ApoA level is significantly lower in women with IUGR than in normal pregnancies but further studies have yet to be carried out to elucidate the mechanisms involved.

The diastolic blood pressure increases progressively throughout pregnancy and could respond to changes in water compartmentalisation or to an increase in the plasma viscosity following competitive inhibition of the plasminogen endothelial ApoB receptors, which is increased during pregnancy. ${ }^{16}$

Fletcher $e t a l^{17}$ have found changes in plasma fibrinogen in pregnant women which are related to an increase in fibrinogen catabolism initiated by the action of thrombin, and causing fibrinogen-fibrin complexes (high molecular weight fibrin complexes-HMWFC). The levels of HMWFC in pregnant women are greater than in non-pregnant women. ${ }^{17} \mathrm{We}$ know of no similar studies in which catabolism of fibrinogen has been evaluated in pregnancies complicated by IUGR.

We did not measure fibrinogen because we did not have large enough sample volumes although we did determine the difference between plasma and serum viscosities to evaluate the changes in viscosity which are produced by fibrinogen. Pregnancy is associated with a rise in fibrinogen and in factors VII, VIII, IX, X, and XII which has been interpreted as being the result of hormonally induced changes in blood coagulation system function. ${ }^{17}$ The plasma viscosity is significantly greater in pregnant women with IUGR, in whom there is a more pronounced increase in fibrinogen level. ${ }^{18} 19$ 
The difference between the plasma viscosity and serum viscosity could be a good index of changes resulting from the fibrinogen fraction. ${ }^{9}$ Variation coefficients indicating the difference between the viscosities in the two groups in our study were greater than those cited by Ernst $e t a l^{9}$ and reveal a large variability in the relationships between the viscosity and fibrinogen. This could be explained by increased fibrinolytic activity in pregnant women ${ }^{172021}$ which makes the viscosity difference method unreliable under the circumstances.

The comparison between the protein fractions of the normal pregnancies and the IUGR group shows differences only in the $\alpha_{1}$ globulin fraction. HDL have a high electrophoretic mobility in the $\alpha_{1}$ band. Although HDL values were not statistically different in the two groups, a compositional change can be observed in these lipoproteins in the IUGR group, with a decrease in ApoA. The relationship between plasma viscosity and $\alpha_{1}$ globulins in normal pregnancies $^{22}$ disappears in pregnancies with IUGR (figure). The concurrent decrease in ApoA and increase in $A p o B$ in pregnancies with IUGR could partly explain the viscosity changes that occur in this group. The affinity of both ApoB and plasminogen for the same endothelial receptors, ${ }^{16}$ leading to competitive inhibition of fibrinolysis, could explain the increase in plasma viscosity observed in the IUGR group.

Causes of the decrease in ApoB and the increase in the other protein fractions within the $\alpha_{1}$ electrophoretic band in pregnant women with IUGR have yet to be discovered. On the basis of our results we suggest that haemorheological modifications in the IUGR group, reported by several investigators, ${ }^{17182324}$ are in part secondary to changes in high density lipoprotein metabolism and the competitive inhibition of fibrinolysis by $A p o B$, which are increased in the pregnant women with IUGR. Although more thorough clinical assays are necessary, changes in ApoA, and more specifically in the ApoB/ApoA ratio, could be good markers for the early detection of IUGR.

We wish to thank Dr A Puertas and the Obstetrics Unit (Virgen de las Nieves Hospital) for technical assistance. We thank Ms Ana Gómez and Ms Caroline Coope for translating the origina manuscript into English.
1 Hay WW. Energy and substrate requirements of the placenta and fetus. Proc Nutr Soc 1991;50:321-36.

2 Thompson A, Billewicz W, Hytten F. The assessment of fetal growth. F Obst Gynaecol Br Cwlth 1968;75:903-9.

3 Allain CL, Poon CJ, Chan CSG, Richmond WW, Fu PL. Enzymatic determination of total serum cholesterol. Clin Chem 1974;20:470-6.

4 Lopes-Virella MF, Stone PG, Ellis S, Colwell JA. Cholesterol determination in high density lipoproteins separated by three different methods. Clin Chem 1977;23:882-6.

5 Brown DF. Blood lipids and lipoproteins in atherogenesis. Am ₹ Med 1969;46:691-704.

6 Takayama MN, Itoh S, Nagasaki T, Tinimizu I. A new enzymatic method for determination of serum choline containing phospholipids. Clin Chim Acta 1977;79:93-8.

7 Friedwald WT, Levy RI, Frederickson DS. Estimation of plasma low density lipoprotein cholesterol concentration without use of the preparative ultracentrifugation. Clin Chem 1972;18:499-509.

8 Hudson L, Fay FC. Interacción del anticuerpo con el antígeno. In: Hudson L, Fay FC, eds. Immunologia Práctica. Barcelona: Jims, 1978:119-21.

9 Enst E, Resch KL, Saradeth T, Maier A, Matrai A. A viscosimetric method of measuring plasma fibrinogen concentrations. F Clin Pathol 1992;45:534-5.

10 Piechota W, Staszewski A. Reference ranges of lipids and apolipoproteins in pregnancy. Eur $\mathcal{F}$ Obstet Gynecol Rep Biol 1992;4527-35.

11 Jimenez DM, Pocovi M, Ramon-Cajal J. Longitudal study of plasma lipids and lipoprotein cholesterol in normal pregnancy and puerperium. Gynecol Obstet Invest 1988; 25:158-64.

12 Potter JM, Nestel PJ. The hyperlipidemia of pregnancy in normal and complicated pregnancies. Am $\mathcal{F}$ Obstet Gynecol normal and complica

13 Darmday JM, Postle AD. Lipid metabolism in pregnancy. Br $\mathcal{F}$ Obstet Gynaecol 1982;89:211-5.

14 Fahreus L, Larsson-Cohn U, Wallentin L. Plasma lipoproteins including high density lipoprotein subfractions during normal pregnancy. Obstet Gynecol 1985;66:468-72.

15 Hillman L, Schonfeld G, Miller JP. Apolipoproteins in human pregnancy. Metabolism 1975;24:943-52.

16 Koenig W, Hehr R, Ditschuneit HH, Kuhn K, Ernst E, Rosenthal J, et al. Lovastatin alters blood rheology in primary hyperlipoproteinemia: dependence on lipoprimary hyperlipoproteinemia: dependence

17 Fletcher AP, Alkjaersig NK, Burstein R. The influence of pregnancy upon blood coagulation and plasma fibrinolytic enzyme function. Am $\mathcal{f}$ Obstet Gynecol 1979;134:743-51.

18 Huisman A, Aarnoudse JG, Heuvelmans JHA, Goslinga H Fidler V, Huisjes HJ, et al. Whole blood viscosity during normal pregnancy. Br $¥$ Obstet Gynaecol 1987;94:1143-90.

19 Pickart LR, Creasy RK, Thaler MM. Hyperfibrinogenemia and polycythemia with intrauterine growth retardation in fetal lambs. Am $\mathcal{F}$ Obstet Gynecol 1976;124:268-71.

20 Thorburn J, Drummond MM, Whigham KA, Lowe GDO, Forbes CD, Prentice CRM, et al. Blood viscosity and haemostatic factors in late pregnancy, pre-eclampsia and fetal growth retardation. Br ₹ Obstet Gynaecol 1982;89: fetal grow-22.

21 Muñoz A, Uberos J, Bonillo A, Valenzuela A, Puertas A, Narbona $\mathrm{E}$, et al. Plasma and internal erythrocyte viscosity in umbilical artery and vein of premature infants with and without acute asphyxia. Clin Hemorheol 1994;14:75-82.

22 Uberos J, Muñoz A, Puertas A, Valenzuela A, Molina A Rodriguez MT. Análisis comparativo de la viscosidad plasmática durante la gestación y períodos feto-neonatal. Contribución de las fracciones proteicas. An Esp Pediatr 1994;41:187-9.

23 Zondervan HA, Oosting J, Handerman MR, SmorenbergSchoorl ME, Treffers PE. The influence of maternal whole blood viscosity on fetal growth. Eur $₹$ Obstet Gynecol Reprod Biol 1987;25:187-94.

24. Piccaro M, Rastelli D, Lombardo F, Ferrazzani S, Bondoli A, Cagnetta G, et al. Fisiopatologia in gravidanza: quadro reologico e bioumorale nella gravidanza normale e patologica. Minerva Ginecol 1982;34:111-26 